\title{
Finite element analysis of a percutaneous aortic valve stent design
}

\author{
Gideon Praveen Kumar ${ }^{1^{*}}$, Lazar Mathew ${ }^{1}$ \\ ${ }^{1}$ School of Biotechnology, Chemical \& Biomedical Engineering, VIT University, Vellore, India. \\ *Corresponding author: Tel: +919894292348, Email: gidi99_5611@yahoo.co.in
}

Received 15 April 2009; revised 28 April 2009; accepted 30 April 2009.

\begin{abstract}
Aim: This paper discusses the design and Finite Element Analysis (FEA) of a Percutaneous Aortic Valve Stent. The aim of this study was to model a percutaneous aortic valve stent and subject it to finite element analysis. The design process was carried out to meet the functional and surgical requirements. Methods and Results: Analysis was done with different materials with loads ranging from $50 \mathrm{kgf} / \mathrm{mm}^{2}$ to $73 \mathrm{kgf} / \mathrm{mm}^{2}$. These forces were selected because these values are far greater than the normal human blood pressure which ranges from $10 \mathrm{kPa}$ to $16 \mathrm{kPa}$. It was also to understand the mechanical behavior of different stent materials under such high pressures. A stent model was generated and its physical, mechanical and behavioral properties were studied. Finite element analysis and simulation of the model enhanced the designer to optimize the geometry suitable for performance during and after implantation. The design objective for the stent is to have long term durability, low thrombogenicity, resistance to migration and paravalvular leak. Conclusion: The analysis performed in this paper may aid in understanding the stent's tolerable pressures ranges in comparison with the physiological pressures exerted by the heart and cardiac blood flow during abnormal cardiovascular conditions.
\end{abstract}

Keywords: stent; finite element analysis; blood flow; aortic valve stenosis; port size

\section{INTRODUCTION}

The treatment of stenotic valvular diseases consists of routine procedures in interventional cardiology. To date, surgical approach is the only option to replace diseased cardiac valves. Recently stenosis of mitral, aortic or pulmonary valves is treated by percutaneous valve re- placement and has opened new perspectives on transcatheter placement of cardiac valves. Aortic valve replacement was generally accomplished by using open heart valve surgery, whereas endovascular procedures for valve replacement may provide an alternative to cardiac surgery. Such endovascular procedures require minimal invasion of the human body, and there is considerable reduction and, in some instances, even elimination of general anesthesia and intensive care unit stay. In Percutaneous replacement, the diseased valve is replaced by a biological valve (porcine) via a catheter driven through the femoral artery to the aortic position. The valve is mounted on the stent, which is crimped into the catheter and is guided to the parent position of the aorta and deployed. Thus it pushes the diseased valve aside to position itself. Post operative complications are very minimal and the patient can get discharged within days after the procedure. The stents that are used give adequate support and stability to the valve and also prevent the valve from getting migrated either in the ante grade or retro grade direction. These stents also help in preventing paravalvular leaks. So modeling of an ideal stent design was done and the subjected to finite element analysis with loads much greater than the expected blood pressures even during adverse conditions.

\section{STENT DESIGN}

The design of the stent depends on many unique parameters, which include stent length, stent diameter, number of struts, strut diameter and port angle. The design was an attempt to model a stent which would be an ideal partner to the tissue valve that replaces the diseased one [1]. Solid 3D models (Figure 1) were created using repeating unit geometry of each design using Solidworks Modeling Software. The unit consisted of 8 lips with two non crossing struts making a circular diameter of $16 \mathrm{~mm}$. Two struts join to form a lip creating a diamond shaped port. The port angle is 45 degrees and the distance between the two struts at the center is $6.25 \mathrm{~mm}$, with 8 lips joining at the center to form the circular stent. The stent design has a constant strut thickness of $0.5 \mathrm{~mm}$ and a height of $18 \mathrm{~mm}$. 

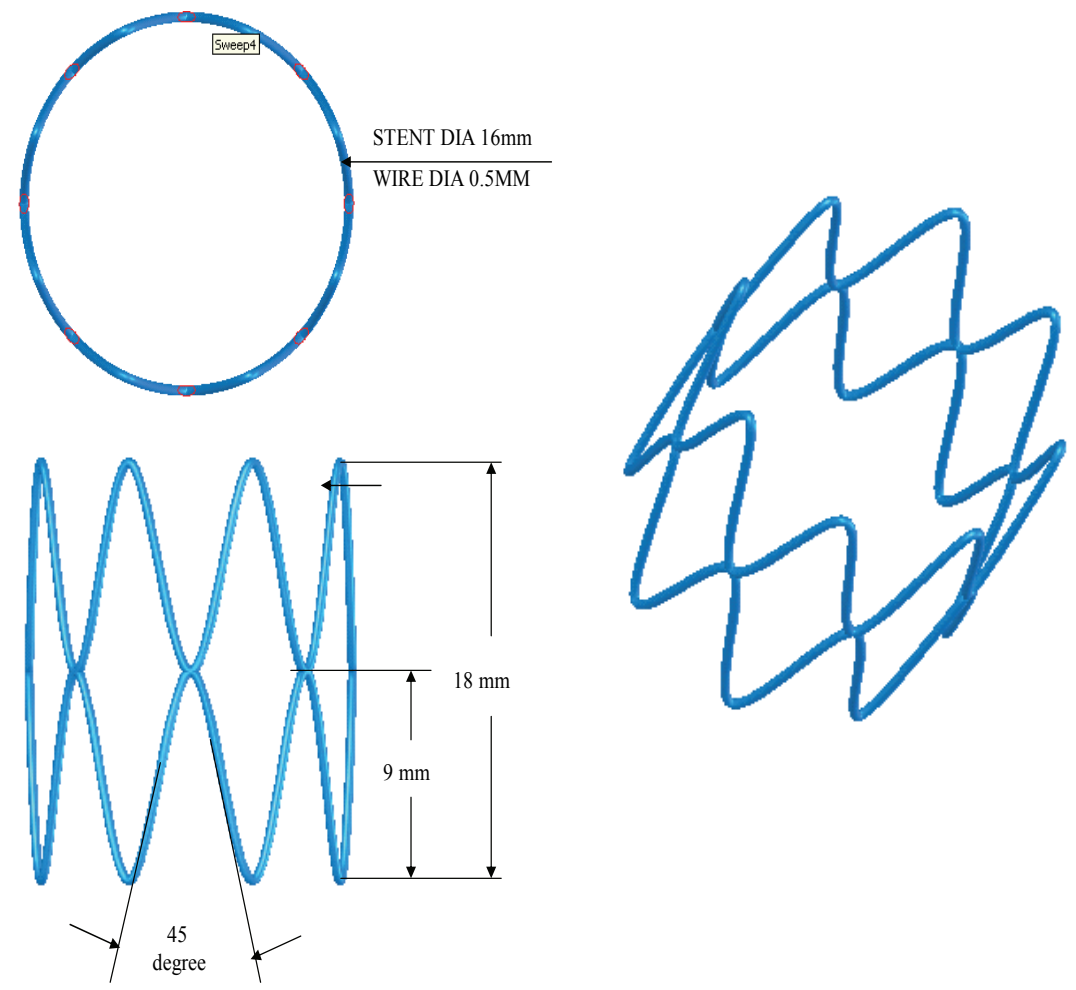

Figure 1. Model geometry of the stent.

\section{MATERIAL MODEL}

Stainless steel $316 \mathrm{~L}$ was the material that was used for analysis as it has a high strength to weight ratio and excellent biocompatibility when used in humans [6]. The material properties ideal for analysis are tabulated in Table 1. The material properties were assumed to be bilinear, elasto-plastic model with isotropic hardening. All materials were also assumed to follow Hooke's Law, and frictional forces neglected due to the magnitude of this force being much less than the applied force. The implant was assumed to transmit all absorbed energy to the surrounding environment. The amount of energy transfer to and from the implant is zero. The effects due to body heat were also neglected to optimize effects.

\section{FINITE ELEMENT ANALYSIS}

Finite element analysis with 316L stainless steel was performed using visual Nastran, a FEA package. Visual Nastran, a powerful general purpose finite element analysis solution for small to complex assemblies, was used for the entire analysis [6]. Stresses ranging between $50 \mathrm{kgf} / \mathrm{mm}^{2}$ and $73 \mathrm{kgf} / \mathrm{mm}^{2}$ were loaded on to the stent in the transverse direction. These pressures were selected as they are far greater than that of the normal human blood pressure which ranges from $10 \mathrm{kPa}$ to $16 \mathrm{kPa}$. Ini-
Table 1. List of properties used for analysis.

\begin{tabular}{lc}
\hline Material Name & 316 L Stainless Steel \\
Young's modulus (E) & $201 \mathrm{GPa}$ \\
Poisson's ratio & 0.3 \\
Yield stress & $170 \mathrm{MPa}$ \\
\hline
\end{tabular}

tially the loads were given as a centripetal pressure around the circular stent in the transverse direction. The reason for choosing this direction is because of the fact that the pulsatile contraction of the aorta is in the same direction. The model was constrained towards one end of the stent. Owing to the direction of load application and the stent being constrained towards one end, the stent was able to crimp and re-expand without stent fracture even when the load applied was far greater than that of the normal physiological pressures. The model was then constrained towards the lower end of the stent. The upper portion of the stent is somewhat free and is along the longitudinal axis of the ascending aorta and the lower portion bears the aortic valve and is fixed to the aortic annulus. Similar pressures were applied in the upward direction to understand the mechanical and physical behavior of the stent. In the same position, pressures were applied in the downward direction to understand the behavior 
of the stent.

\section{RESULTS AND DISCUSSION}

The stent design was subjected to finite element analysis and its mechanical behavior was studied. The load for all the analyses ranged from $50 \mathrm{kgf} / \mathrm{mm}^{2}$ to $73 \mathrm{kgf} / \mathrm{mm}^{2}$.
Table 2. Displacement for load in the crimped stent model.

\begin{tabular}{cc}
\hline Force $\left(\right.$ kgforce $\left./ \mathrm{mm}^{2}\right)$ & Displacement \\
72.1 & 0.36 \\
60.7 & 0.28 \\
57.3 & 0.13 \\
49.6 & 0.09 \\
\hline
\end{tabular}

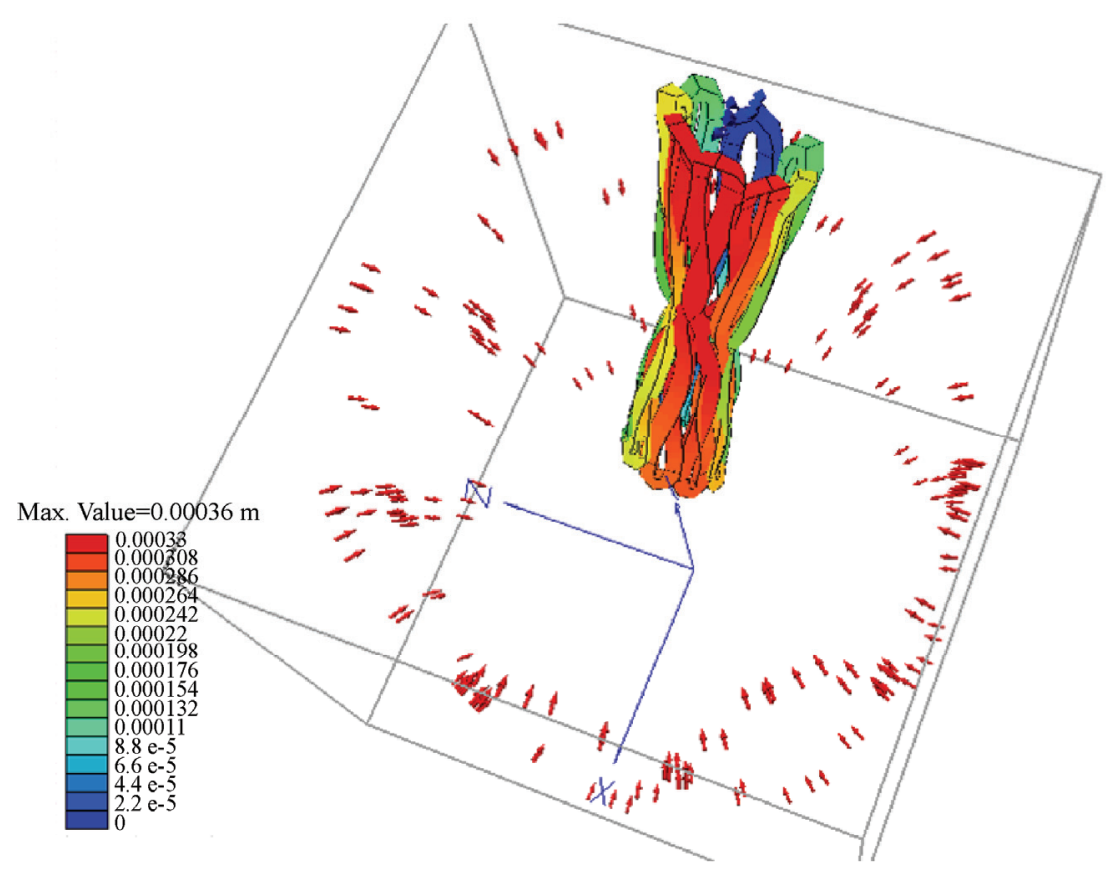

Figure 2. Displaced shape of the stent.

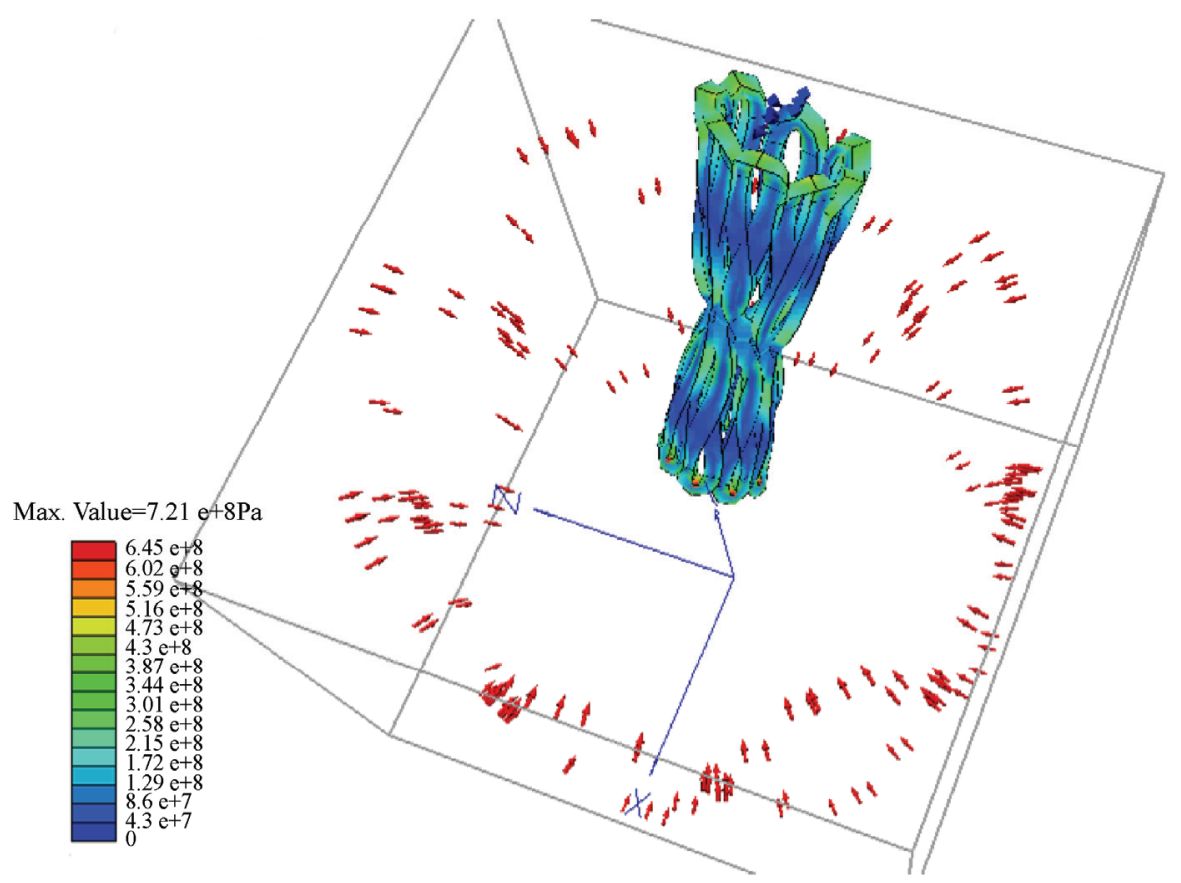

Figure 3. Equivalent stress contours during crimping. 
A contour plot of the equivalent von Mises stress shows the location of the maximum stress is represented in Figures 3, 5 and 6. Correlative displacements of the stent during various positions are represented in Figures 2, 4 and 7. The maximum stresses applied to the stent, and its displacements in different positions were compared. It can be noted that the stent was able to withstand loads that were far greater than the stress that may be exerted by the heart and blood even during pathological conditions. Table 2 gives the displacement for the given load on the stent model which is fully crimped and Table 3 gives the displacement for the given load on the stent model which is fixed at the bottom.
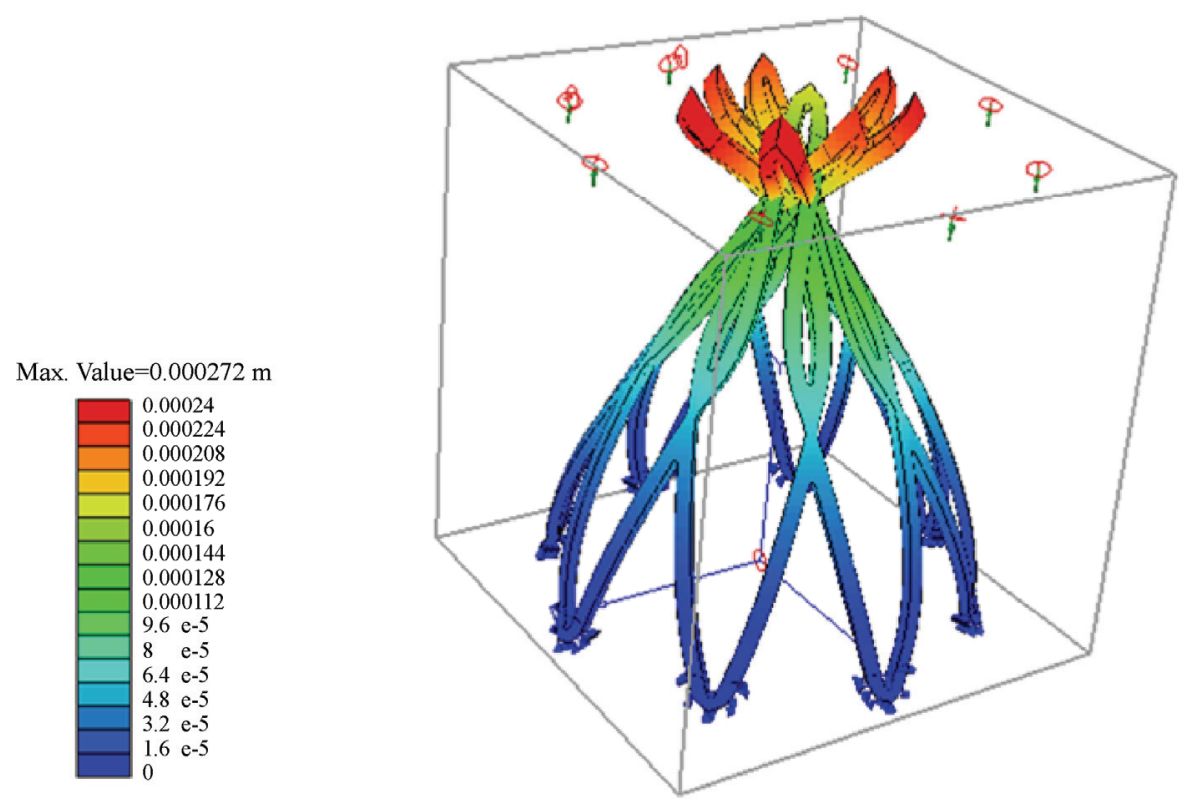

Figure 4. Displaced shape with the stent constrained towards the lower end.

Max. Value $=5.35 \mathrm{e}+8 \mathrm{~Pa}$

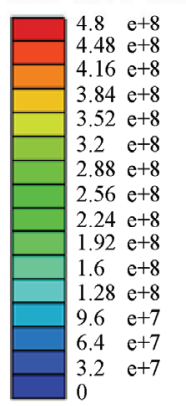

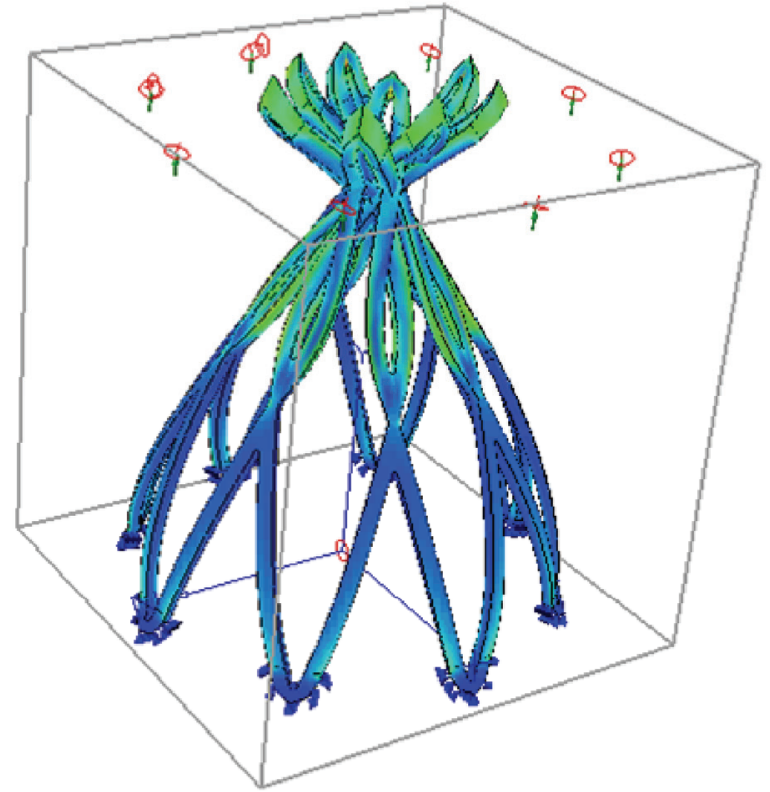

Figure 5. Equivalent stress contours with the stent constrained towards the lower end. 
Defomations scaled by 44.3

Load Factor-1

TOP EDGE(8 LIPS)

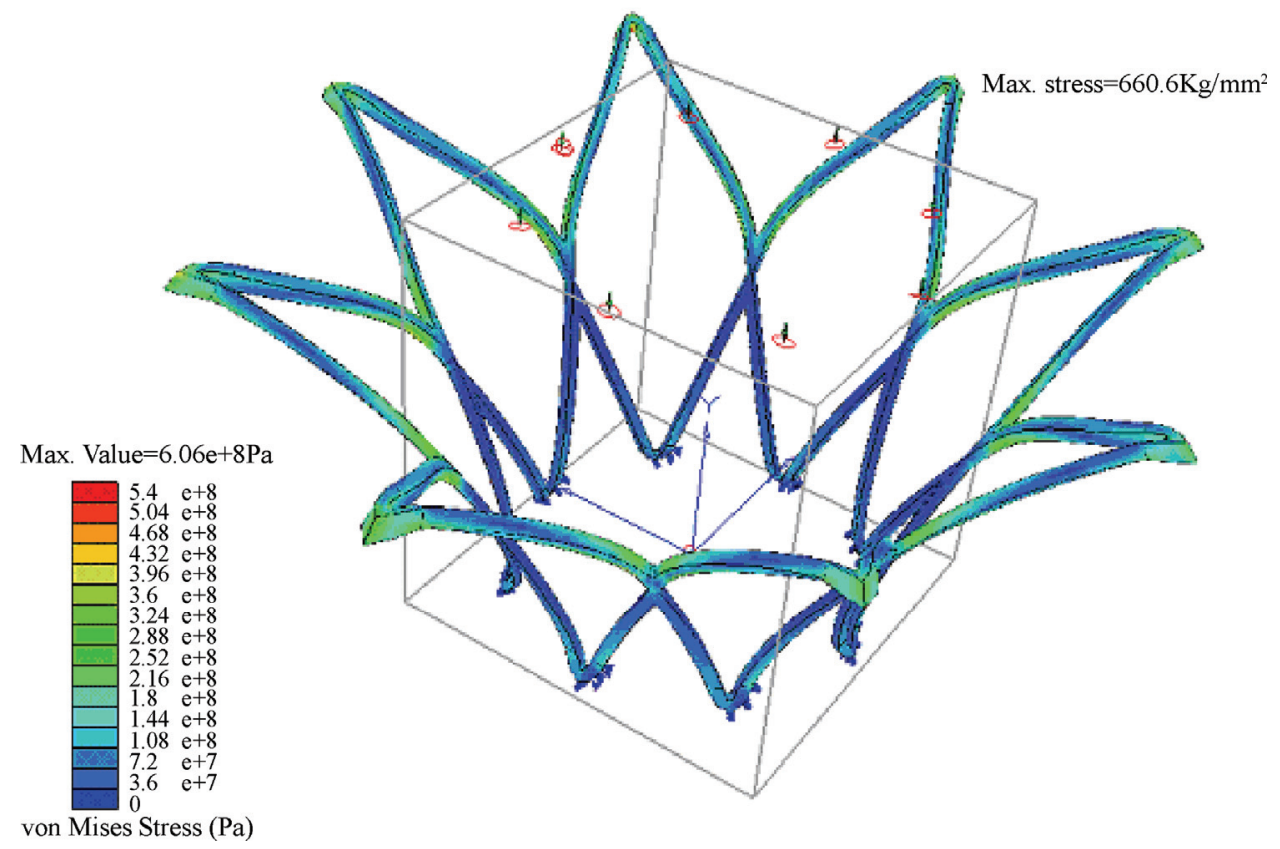

Figure 6. Equivalent stress contours when a force in the downward direction is applied.

Deformations scaled by 293

Load Factor $=1$

Max. Value $=2.56 \mathrm{e}-5 \mathrm{~m}$
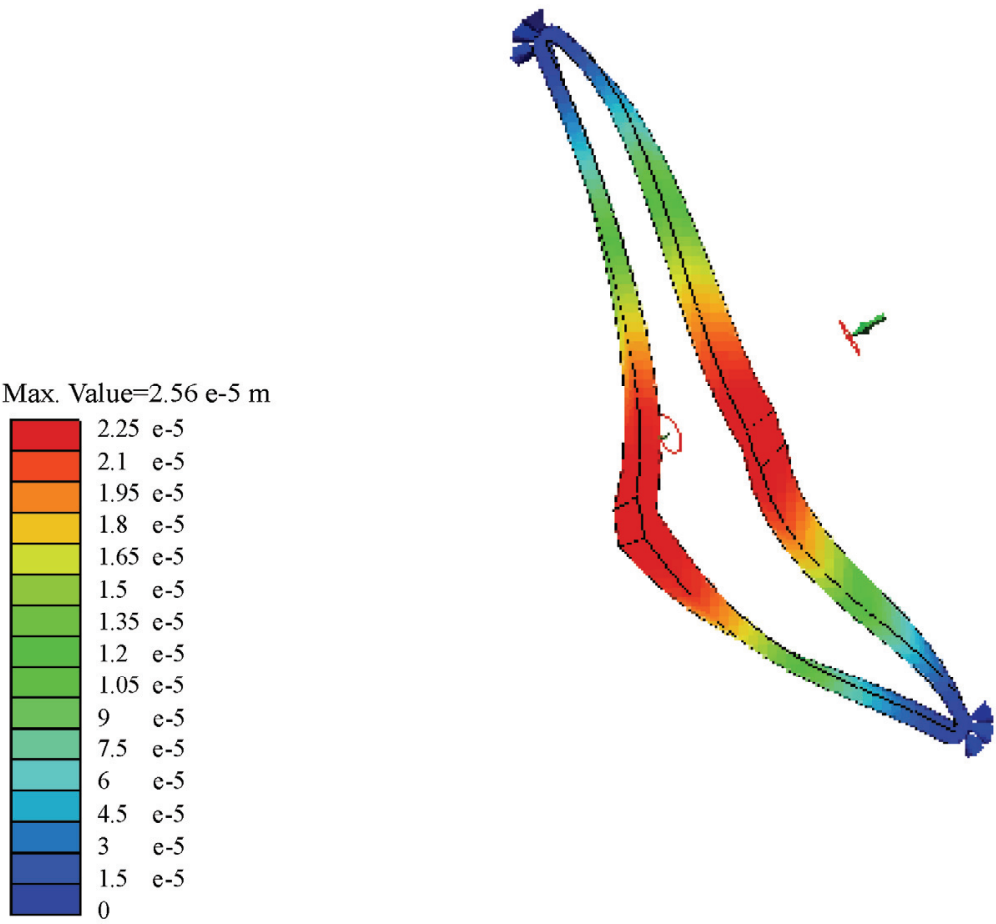

Delta_MAG Displacement (m)

Figure 7. Displaced shape of a single lip. 
Table 3. Displacement for load in the stent model fixed at the bottom.

\begin{tabular}{cc}
\hline $\begin{array}{c}\text { Force } \\
\left(\mathrm{kgforce} / \mathrm{mm}^{2}\right)\end{array}$ & $\begin{array}{c}\text { Displacement } \\
(\mathrm{mm})\end{array}$ \\
\hline 53.5 & 0.27 \\
47.2 & 0.22 \\
43.7 & 0.16 \\
38.1 & 0.11 \\
\hline
\end{tabular}

\section{CONCLUSION}

The stent design modeled and subjected to Finite element analysis proved to be having ideal behavior for percutaneous replacement by withstanding loads that are well above the required level. The displacement was good enough to prove mild contractions during cardiac systole and diastole.

\section{REFERENCES}

[1] C. M. Allison, D. Crossman, and J. Gunn, (2004) The influence of physical stent parameters upon restenosis, Elsevier SAS.

[2] K. W. Lau, A. Johan, U. Sigwart, and J. S. Hung, (2004)
A stent is not just a stent: stent construction and design do matter in its clinical performance, Singapore Medical Journal.

[3] D. R. McClean and N. Eigler, (2002) Stent design: Implications for restenosis, MedReviews, LLC.

[4] (2004) Stent design properties and deployment ratio influence indexes of wall shear stress: A three-dimensional computational fluid dynamics investigation within a normal artery, J Appl Physiol, 97, 424-430.

[5] (2000) An overview of superelastic stent design, Min Invas Ther \& Allied Technol, 9(3/4), 235-246.

[6] (2007) Engineering aspects of stents design and their translation into clinical practice, Ann Ist super sAnItà, 43(1), 89-100.

[7] (2005) To stent or not to stent aortic dissection:good news for a chosen few, but who? European Heart Journal, 26, 431-432, doi:10.1093/eurheartj/ehi119.

[8] J. Q. Zhou, A. F. Corno, C. H. Huber, P. Tozzi, and L. K. von Segesser, (2003) Self-expandable valved stent of large size: off-bypass implantation in pulmonary position, Eur. J. Cardiothorac. Surg., 24(2), 212-216.

[9] G. P. Kumar and L. Mathew, (2009) New stent design for percutaneous aortic valve replacement, International Journal of Cardiovascular Revascularization Medicine, 10(2), 121-124. 